\title{
Triiodothyronine Reduces Vascular Dysfunction Associated with Hypertension by Attenuating Protein Kinase G/Vasodilator-Stimulated Phosphoprotein Signaling ${ }^{[}$
}

\author{
Maria Alicia Carrillo-Sepulveda, Anjali Panackal, Renjith Maracheril, Nicole Maddie, \\ Mitul N. Patel, Kaie Ojamaa, Olga V. Savinova, and A. Martin Gerdes \\ Department of Biomedical Sciences, New York Institute of Technology, College of Osteopathic Medicine, Old Westbury, \\ New York
}

Received May 31, 2019; accepted July 9, 2019

\begin{abstract}
Vascular dysfunction associated with hypertension comprises hypercontractility and impaired vasodilation. We have previously demonstrated that triiodothyronine (T3), the active form of thyroid hormone, has vasodilatory effects acting through rapid onset mechanisms. In the present study, we examined whether T3 mitigates vascular dysfunction associated with hypertension. To test the direct effects of T3 in hypertensive vessels, aortas from female Dahl salt-sensitive (Dahl SS) rats fed a high-salt diet ( $8 \% \mathrm{NaCl}, \mathrm{HS}$ group) and their age-matched controls fed a standard low-salt diet $(0.3 \% \mathrm{NaCl}, \mathrm{LS}$ group) for 16 weeks were isolated and used in ex vivo vascular reactivity studies. We confirmed that the HS group exhibited a higher systolic blood pressure in comparison with the control LS group and displayed aortic remodeling. Aortas from both groups were pretreated with T3 $(0.1 \mu \mathrm{M})$ for 30 minutes at $37^{\circ} \mathrm{C}$ in a $5 \% \mathrm{CO}_{2}$ incubator before functional vascular studies. T3 treatment significantly attenuated hypercontractility and improved impaired endothelium-dependent vasodilation in aortas from the HS group. These vascular improvements in response to T3 were accompanied by increased phosphorylation of vasodilator-
\end{abstract}

stimulated phosphoprotein (VASP) at serine 239, a vasodilatory factor of the cGMP-dependent protein kinase (PKG)/ VASP signaling pathway in vascular smooth muscle cells. Moreover, increased production of reactive oxygen species in aortas from the HS group were significantly reduced by T3, suggesting a potential antioxidant effect of T3 in the vasculature. These results demonstrate that T3 can mitigate hypertension-related vascular dysfunction through the VASP signaling pathway and by reducing vascular ROS production.

\section{SIGNIFICANCE STATEMENT}

This study demonstrates that triiodothyronine (T3) directly acts on vascular tone and has a beneficial effect in hypertensioninduced vascular dysfunction. T3 augmented vasodilation and diminished vasoconstriction in blood vessels from hypertensive rats in association with activation of the protein kinase G/vasodilator-stimulated phosphoprotein signaling pathway that activates vascular relaxation and exerted an antioxidant effect. Collectively, these results show that T3 is a potential vasoprotective agent with rapid action on hypertensionrelated vascular dysfunction.

\section{Introduction}

Hypertension is a multifactorial disease characterized by an elevation of arterial blood pressure, which progressively increases the risk for cardiovascular disease (Mancia and Giannattasio, 2015). Reduction in vasodilation and increased vasoconstriction characterize vascular dysfunction in essential hypertension (Mordi et al., 2016). Thyroid hormones have also been identified as having an important role in maintaining vascular tone (Ojamaa et al., 1993, 1996; Park et al., 1997; Khorshidi-Behzadi et al., 2013). An alteration of circulating levels of thyroid hormones can negatively affect homeostasis and vascular function (Khorshidi-Behzadi et al., 2013). In this

https://doi.org/10.1124/jpet.119.260471.

S This article has supplemental material available at jpet.aspetjournals.org. regard, a strong association between thyroid hormone dysregulation and hypertension has been reported (Rapp, 1982). Using spontaneously hypertensive heart failure (SHHF) rats, our group reported that, although significant thyroid dysfunction is present in younger hypertensive animals, long-term treatment with a low dose of thyroid hormone can attenuate cardiac dysfunction in SHHF rats (Kisso et al., 2008; Weltman et al., 2015).

Among the experimental models of hypertension available, the Dahl salt sensitive (Dahl SS) rat strain is a genetic model of human hypertension that displays vascular dysfunction (Drenjancevic-Peric and Lombard, 2004; Teran et al., 2005) and rapid progression of hypertension in response to a highsalt diet (Rapp, 1982; Sullivan, 1991). However, the role of triiodothyronine (T3) in vascular dysfunction associated with hypertension in Dahl SS rats remains undetermined. T3 is

ABBREVIATIONS: ACh, acetylcholine; Dahl SS rats, Dahl salt sensitive rats; DHE, dihydroethidium; HS, high-salt diet; LS, low-salt diet; NO, nitric oxide; PE, phelynephrine; PKG, protein kinase G; ROS, reactive oxygen species; SHHF, spontaneously hypertensive heart failure; T3, triiodothyronine; VASP, vasodilator-stimulated phosphoprotein; VSMC, vascular smooth muscle cell. 
known to cause rapid vascular relaxation and likely plays a crucial role in vascular homeostasis (Samuel et al., 2017). Our group has recently identified that T3 improves vascular function via a nongenomic mechanism involving the protein kinase G/vasodilator-stimulated phosphoprotein (PKG/VASP) signaling pathway (Samuel et al., 2017). In the current study, we determined whether T3 can improve vascular function in Dahl SS rats. The findings of this study provide evidence that T3 is a potent vasoactive factor that improves hypertensive vascular dysfunction.

\section{Materials and Methods}

Animal Model. Dahl SS rats were obtained from Harlan Laboratories (Indianapolis, IN). Eight-week-old female Dahl SS rats were randomly assigned to a standard low-salt diet $(n=7)$ containing $0.3 \% \mathrm{NaCl}$ (LS group, D10101402; Research Diets, New Brunswick, $\mathrm{NJ})$ or a high-salt $\operatorname{diet}(n=7)$ containing $8 \% \mathrm{NaCl}$ (HS group, D10101401; Research Diets) for 16 weeks. Rats were housed in a temperature-controlled room with a 12-hour light/dark cycle, and were allowed food and water ad libitium.

At the end of the experimental protocol, the rats were anesthetized with an inhalation of $5 \%$ isoflurane in $100 \%$ oxygen. A thoracotomy was then performed, and blood was obtained from the right ventricle and serum was collected for T3 analysis. Thoracic aortas were harvested for immediate analysis of vascular reactivity and other parameters under T3 treatment conditions. All experimental protocols were conducted in accordance with the Institutional Animal Care and Use Committee at New York Institute of Technology College of Osteopathic Medicine and with respect to the National Institutes of Health (NIH, Publication N.85-23, revised 2010) Guidelines for the Care and Use of Laboratory Animals.

Blood Pressure Measurement. Blood pressure was measured in conscious restrained rats using CODA High Throughput Noninvasive Blood Pressure System and analyzed with CODA Version 4.1 software (Kent Scientific Corporation, Torrington, CT) according to the manufacturer's protocols. The rats were pretrained/acclimated to the apparatus before pressure recordings. Twenty (20) measurements of blood pressure were averaged. Blood pressure was measured between $9 \mathrm{AM}$ and $12 \mathrm{PM}$.

Serum T3 Measurements. Blood was collected from the right ventricle and left to clot at room temperature for 30 minutes and then centrifuged at $2800 \mathrm{rpm}$ for 15 minutes. Serum was immediately aliquoted and stored at $-80^{\circ} \mathrm{C}$ until assayed for total $\mathrm{T} 3$, using an ELISA kit (Monobind, Lake Forest, CA) according to the manufacturer's instructions.

Aortic Histomorphometric Analysis. Cryosections of aortas $(5 \mu \mathrm{m})$ were mounted on slides and fixed in $10 \%$ formalin and stained with H\&E (Sigma-Aldrich, St. Louis, MO). High-resolution bright field and fluorescent light digital images were captured using an Olympus IX71 microscope equipped with a DP73 camera. Morphometric analysis including cross-sectional area and wall-to-lumen ratio of the aortas were obtained using ImageJ software (National Institutes of Health, Bethesda, MD) according to established protocols (Gómez-Roso et al., 2009)(; Maia et al., 2014).

Vascular Reactivity. Isolated thoracic aortas were incubated in oxygenated Krebs buffer $(130 \mathrm{mM} \mathrm{NaCl}, 14.9 \mathrm{mM} \mathrm{NaHCO}$, $4.7 \mathrm{mM} \mathrm{KCl}, 1.18 \mathrm{mM} \mathrm{KH}_{2} \mathrm{PO}_{4}, 1.17 \mathrm{mM} \mathrm{MgSO}_{4}-7 \mathrm{H}_{2} \mathrm{O}, 1.56 \mathrm{mM}$ $\mathrm{CaCl}_{2}-2 \mathrm{H}_{2} \mathrm{O}, 0.026 \mathrm{mM}$ EDTA, $5.5 \mathrm{mM}$ glucose, $\mathrm{pH}$ 7.4), with the perivascular fat carefully removed. The aortas were cut into rings ( $2 \mathrm{~mm}$ in length) and cultured in vascular medium (American Type Culture Collection [ATCC], Manassas, VA) in an incubator at $37^{\circ} \mathrm{C}$ supplied by $5 \% \mathrm{CO}_{2}$. The aortas were treated with $0.1 \mu \mathrm{M}$ T3 (Sigma-Aldrich) for 30 minutes. The concentration of T3 was chosen based on results from a previous study (Samuel et al., 2017). A stock solution of T3 was made by dissolving T3 in DMSO (ATCC) and further diluted in Krebs solution. A DMSO vehicle was used in controls.

After treatment, the aortic rings were mounted in a Multi-Wire Myograph System 620M (Danish Myo Technology, Aarhus, Denmark) for isometric tension recordings using a PowerLab 8/35 data acquisition system (ADInstruments Pty Ltd., Castle Hill, Australia). The aortic rings were equilibrated in Krebs buffer for 30 minutes, and in chambers perfused with $5 \% \mathrm{CO}_{2}$ in $95 \% \mathrm{O}_{2}$ at $37^{\circ} \mathrm{C}$, as we have previously described elsewhere (Carrillo-Sepulveda et al., 2013). In all experiments, aortic ring integrity was assessed by stimulation with $120 \mathrm{mM} \mathrm{KCl}\left(74.7 \mathrm{mM} \mathrm{NaCl}, 14.9 \mathrm{mM} \mathrm{NaHCO}_{3}, 60 \mathrm{mM} \mathrm{KCl}, 1.18 \mathrm{mM}\right.$, $\mathrm{KH}_{2} \mathrm{PO}_{4}, 1.17 \mathrm{mM} \mathrm{MgSO}{ }_{4}-7 \mathrm{H}_{2} \mathrm{O}, 1.6 \mathrm{mM} \mathrm{CaCl}{ }_{2}-2 \mathrm{H}_{2} \mathrm{O}, 0.026 \mathrm{mM}$ EDTA, $5.5 \mathrm{mM}$ glucose).

To test for the presence of endothelium, segments were contracted with $1 \mu \mathrm{M}$ phelynephrine (PE) (Sigma-Aldrich); once the vessels reached a stable maximum tension, the vessels were stimulated with $10 \mu \mathrm{M}$ acetylcholine (ACh) (Sigma-Aldrich), and relaxation was confirmed. For the stock solution, $\mathrm{PE}$ and $\mathrm{ACh}$ were dissolved in distilled water. Aortic rings that achieved relaxation to ACh were considered to have a preserved endothelium. Cumulative concentration-response curves to ACh $(1 \mathrm{nM}$ to $10 \mu \mathrm{M})$ and $\mathrm{PE}$ ( $1 \mathrm{nM}$ to $10 \mu \mathrm{M}$ ) were performed on intact aortic rings in the absence or presence of $0.1 \mu \mathrm{M}$ T3. Endothelium-dependent relaxation was recorded for ACh after maximal precontraction with $1 \mu \mathrm{M}$ PE.

Immunoblotting. Protein expression was analyzed by Western blotting. Total protein was extracted from the aortas of HS and LS rats after treatment with $0.1 \mu \mathrm{M}$ T3 for 30 minutes. Equivalent amounts of protein $(40 \mu \mathrm{g})$ were loaded and separated by $10 \%$ SDSPAGE, and transferred to polyvinylidene fluoride membranes (Thermo Fisher Scientific, Rockfold, IL) as previously described elsewhere (Samuel et al., 2017). The membranes were blocked for 1 hour with $5 \%$ nonfat milk solution in Tris-buffered saline with $0.1 \%$ Tween-20 (TBST), then incubated overnight at $4^{\circ} \mathrm{C}$ with the following specific primary antibodies: PKG (1:1000, catalog no.3248; Cell Signaling Techonology, Danvers, MA), total and phosphorylated VASP (1:1000, catalog no. 3132, catalog no. 3114; Cell Signaling Techonology), then followed by anti-IgG-horseradish peroxidase secondary antibodies $(1: 10,000$, catalog no. 7074 , catalog no. 7076; Cell Signaling Technology).

The membranes were stripped and reprobed with an internal loading control, $\beta$-actin (1:10,000, catalog no. 4967; Cell Signaling). Protein bands were detected using Enhanced Chemiluminescence (ECL) Ultra reagents (Lumigen Inc., Southfield, MI). The PKG data are presented as percentage $(\%)$ relative to the LS group after normalization to $\beta$-actin. The phospho-VASP measurements were normalized to total VASP levels and expressed as percentage (\%) relative to the LS group.

Measurement of Reactive Oxygen Species. Global reactive oxygen species (ROS) were detected in aortic tissues using $25 \mu \mathrm{M}$ fluorescent probe dihydroethidium (DHE; Sigma Aldrich) as previously described elsewhere (Carrillo-Sepulveda et al., 2015). Aortic rings were obtained from the HS and LS groups. Briefly, the aortic rings were pretreated with $0.1 \mu \mathrm{M}$ T3 for 30 minutes, embedded in Tissue Tek OCT compound (Sakura Finetek, Terrance, CA), snap frozen, and stored at $-80^{\circ} \mathrm{C}$. Cryosections of $5-\mu \mathrm{m}$ thickness were cut and placed on Superfrost Plus slides (Menzel-Gläzer, Braunschweig, Germany). Fifty microliters of DHE solution was applied to each tissue section and then covered with a coverslip. The slides were incubated in a light-protected humidified chamber at $37^{\circ} \mathrm{C}$ for 30 minutes before imaging.

Fluorescence of DHE was detected using an Olympus IX71 fluorescence microscope fitted with a DP73 camera. Quantitative analysis was performed to detect changes in fluorescence using ImageJ software (National Institutes of Health). Background fluorescence was subtracted for each image before quantification. The results are expressed as a percentage (\%) fluorescence in HS and HS + T3 aortic rings in comparison with the LS tissue. 

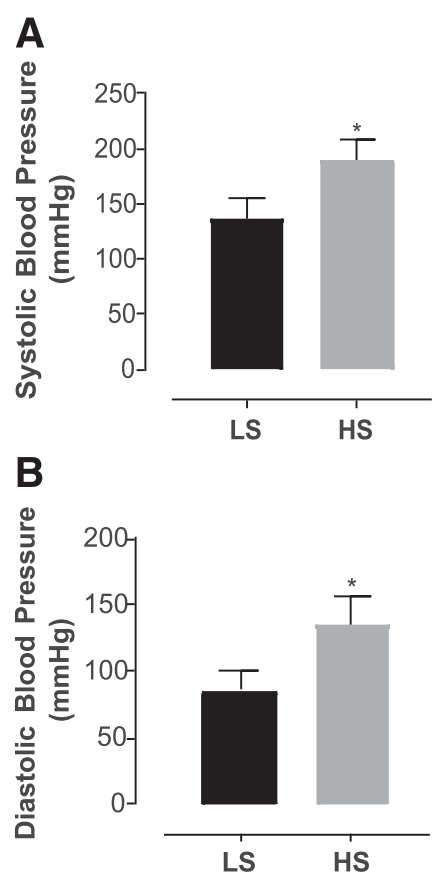

Fig. 1. High-salt diet increased systolic and diastolic blood pressure. Systolic (A) and diastolic (B) blood pressure in Dahl SS rats fed a high-salt $\operatorname{diet}$ (HS group) and low-salt diet (LS group) for 16 weeks measured by tailcuff method. Data are expressed as mean \pm S.E.M.; $n=7$ per group. $* P<$ 0.05 compared with LS group.

Statistical Analysis. Results are expressed as mean \pm S.E.M. All data were analyzed using GraphPad Prism version 7 (GraphPad Software, San Diego, CA). Statistical significance among groups was determined using one-way ANOVA followed by Bonferroni post hoc tests. Student's $t$ test was used when appropriate. $P<0.05$ was considered statistically significant.

\section{Results}

Characterization of Dahl SS Rats. Female Dahl SS rats fed a high-salt diet for 16 weeks (HS group) exhibited increased systolic and diastolic blood pressure in comparison with rats fed a low-salt diet for the same period of time (LS group), as shown in the Fig. 1, A and B, respectively. Concentrations of total T3 in serum of LS and HS rats at the end of the study were not significantly different (1.732 \pm 0.150 and $1.834 \pm 0.335 \mathrm{ng} / \mathrm{ml}$, respectively). In addition to hypertension, the HS group also exhibited marked aortic remodeling, as evident in the histologic sections of thoracic aorta in Fig. 2A. An increased cross-sectional area (Fig. 2B) and greater wall-to-lumen ratio were observed in the HS group compared with the LS group, confirming aortic remodeling (Fig. 2C).

T3 Mitigates Hypertensive Vascular Dysfunction. Vascular dysfunction in hypertensive Dahl SS rats has been well described (Lüscher et al., 1987). To determine whether hypertensive vascular dysfunction is mitigated by shortterm treatment with T3, aortas from the HS and LS groups were isolated and used for vascular reactivity studies. Impaired endothelium-dependent relaxation recorded in aortas from the HS group was attenuated with short-term T3 treatment (Fig. 3A), with the maximal effect generated by the agonist $\left(E_{\max }\right)$ of $46.85 \% \pm 2.90 \%$ versus $63.41 \% \pm$ $4.9 \%$, which is the HS group versus the HS + T3 group, respectively (Fig. 3B). In contrast, T3 did not change relaxation response in aortas from the LS group under the same conditions (Fig. 3, C and D).

Aortas from the HS group showed increased contractility in response to $\mathrm{PE}$ that was significantly reduced with short-term T3 treatment (Fig. 4, A and B). T3 treatment did not alter contractile response in aortas from the LS group (Fig. 4, C and D). Moreover, in vivo experiments

\section{A}

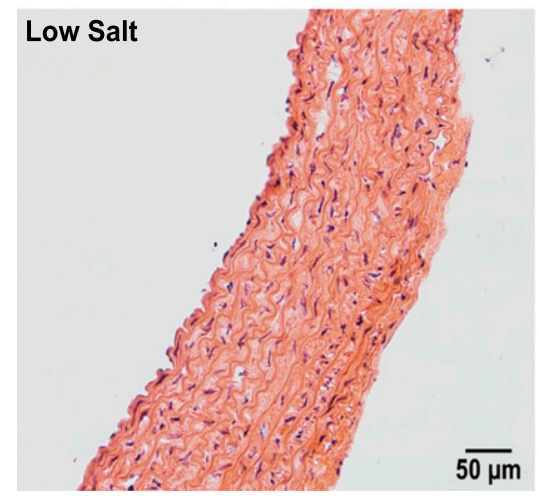

B

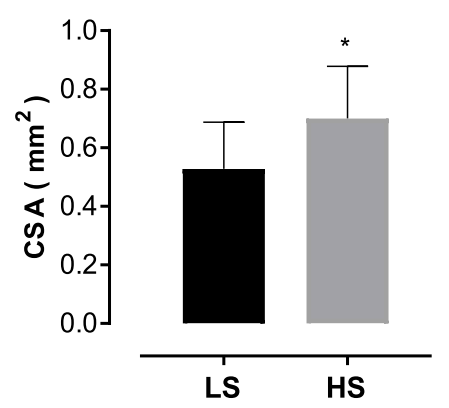

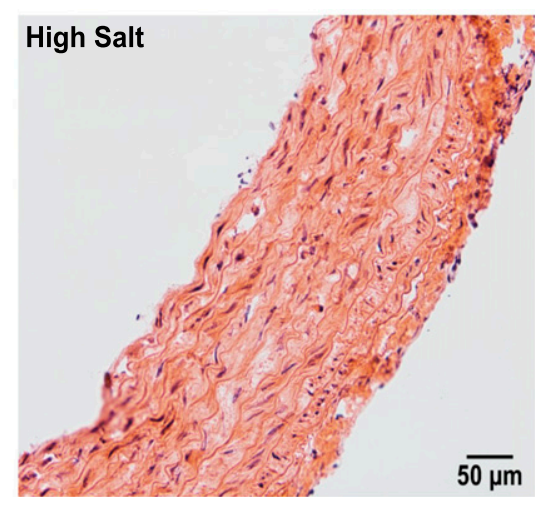

C

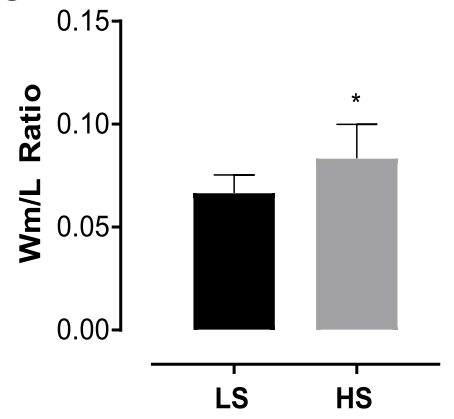

Fig. 2. Aortic remodeling in Dahl SS rats. Representative thoracic aorta cross-sections (A) stained with H\&E. Medial cross-sectional area (B) was increased in the HS group. Wall-to-lumen ratio (C) was increased in the HS group. Data are expressed as mean \pm S.E.M.; $n=8-12$ per group. ${ }^{*} P<0.05$ compared with LS group. 

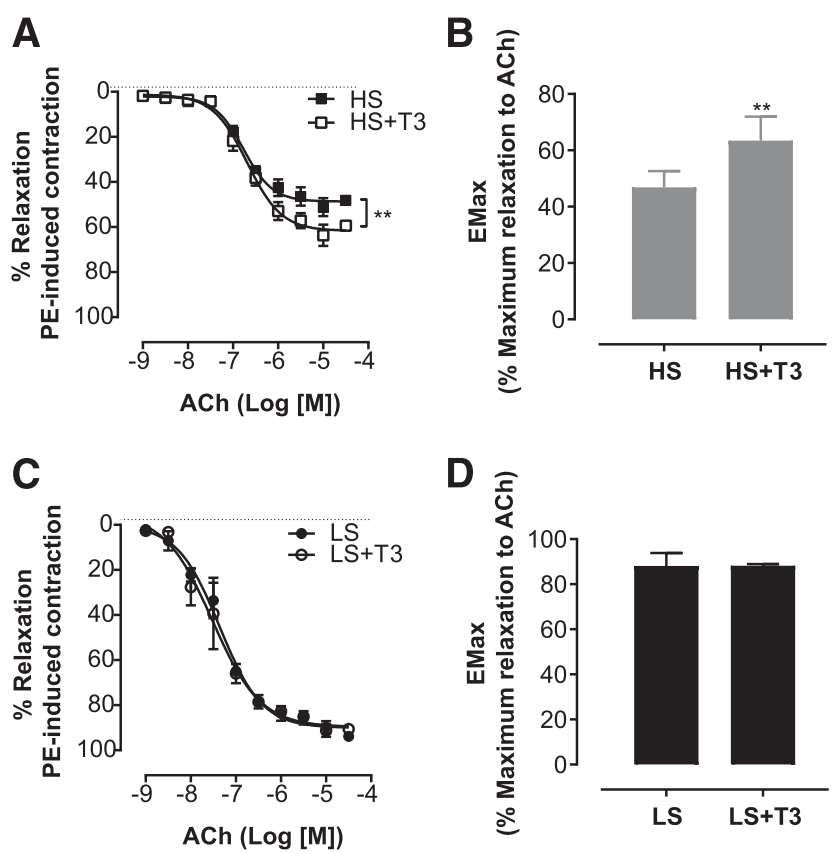

Fig. 3. T3 improved relaxation response in hypertensive aortas. Cumulative concentration curve and maximum response $\left(E_{\max }\right)$ to ACh in aortas from Dahl SS rats fed a high-salt diet (HS group) (A and B) and low-salt diet (LS group) (C and D). Aortas from both groups were pretreated with $0.1 \mu \mathrm{M}$ T3 for 30 minutes (open squares and circles). Data are expressed as mean \pm S.E.M.; $n=7$ per group. ${ }^{*} P<<0.05$ compared with HS group.

showed that rats from HS group treated with T3 $(5 \mu \mathrm{g} / \mathrm{kg}$ per day) in drinking water for 4 weeks (Supplemental Methods) did not display changes in water intake (Supplemental Fig. 1) or blood pressure (Supplemental Fig. 2).

T3 Activates PKG/VASP Signaling in the Aortas of HS-Treated Rats. Based on our previous study showing that T3 promotes vascular relaxation via a PKG/VASP signaling pathway in cultured vascular smooth muscle cells (VSMC) (Samuel et al., 2017), we examined whether improvement of function in hypertensive aortas caused by short-term treatment with T3 is associated with activation of PKG/VASP signaling. The immunoblot analysis revealed that expression of PKG in aortas from the HS group was significantly reduced in comparison with the LS group (Fig. 5). Moreover, levels of phosphorylated VASP, a downstream target of PKG and a marker of vasorelaxation, were decreased in aortas from the HS group as compared with the LS group (Fig. 6). Short-term T3 treatment significantly increased VASP phosphorylation in aortas from HS group (Fig. 6). Together, these results indicate that T3 is able to activate the PKG/VASP signaling pathway in hypertensive vessels.

Effect of T3 on Vascular Oxidative Stress. It has been well-established that oxidative stress is involved in the pathogenesis of hypertensive vascular dysfunction (Touyz and Schiffrin, 2004). To further investigate whether T3 exerts an antioxidant effect in hypertensive vessels, we measured oxidative stress by detecting aortic ROS levels by using DHE staining. The increased production of ROS found in aortas from the HS group was significantly reduced with short-term T3 treatment (Fig. 7).
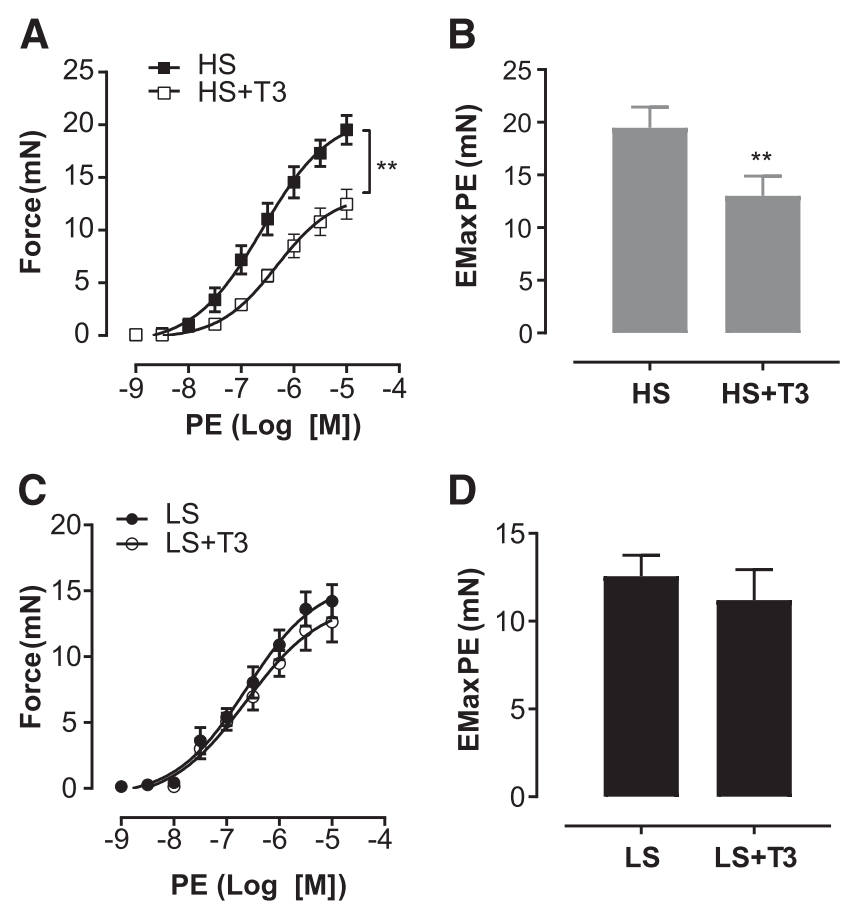

Fig. 4. T3 attenuated augmented contractile response in hypertensive aortas. Cumulative concentration curve and maximum response $\left(E_{\max }\right)$ to $\mathrm{PE}$ in aortas from Dahl SS rats fed a high-salt diet (HS group) (A and B) and low-salt diet (LS group) (C and D). Aortas from both groups were pretreated with $0.1 \mu \mathrm{M}$ T3 for 30 minutes (open squares and circles). Data are expressed as mean \pm S.E.M.; $n=7$ per group. $* * P<0.05$ compared with HS group.

\section{Discussion}

The main findings of the current study are that short-term T3 treatment 1) attenuated vascular dysfunction by increasing vasodilation and diminishing vasoconstriction, 2) activated the vascular PKG/VASP signaling pathway, and 3) reduced oxidative stress in aortas from hypertensive Dahl SS rats. Collectively, these data imply that T3 exerts direct vascular effects and supports T3 as a potential vasoprotective agent for hypertension-related vascular dysfunction.

A series of studies has demonstrated that administration of T3 at pharmacologic concentration causes vascular relaxation (Liu et al., 2014; Samuel et al., 2017). For several years, T3-induced vascular relaxation was attributed to endothelium-dependent mechanisms (Lozano-Cuenca et al., 2016; Razvi et al., 2018), while the VSMC-related mechanisms mediating the vasodilatory effect of T3 remained understudied. In this regard, early work by Ojamaa et al. (1993, 1996) demonstrated that T3 can directly exert its vasodilatory effect through VSMC mechanisms. Consistent with that finding, our group has recently shown that T3 elicits rapid vascular relaxation via activation of PKG/VASP signaling in VSMC (Samuel et al., 2017). Despite growing evidence for participation of both endothelial and VSMC on T3-induced vascular relaxation (Cai et al., 2015), additional studies are needed to fully understand the crosstalk between endothelium-dependent and -independent mechanisms involved in the vascular effects of T3 in different pathophysiologic contexts.

An association between thyroid hormone dysregulation and hypertension has been reported in clinical and basic 

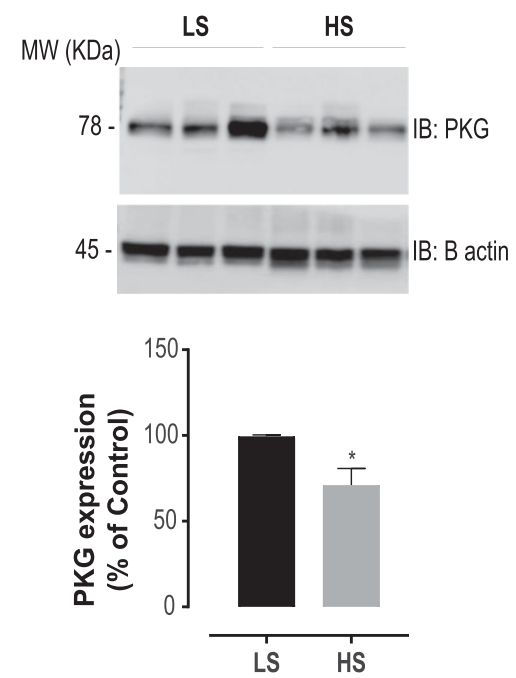

Fig. 5. Decreased aortic PKG expression in Dahl SS rats fed a high-salt diet. Levels of PKG were assessed in aortas from the HS and LS groups using Western blot analysis. Top panel: representative immunoblots. Bottom panel: quantification of intensity of bands. Data are expressed as mean \pm S.E.M., $n=7$ per group, ${ }^{*} P<0.05$ vs. LS group.

experimental studies (Rapp, 1982; Polat Canbolat et al., 2017; Gu et al., 2018). We hypothesized that short-term T3 administration would increase vasodilation and decrease vasoconstriction in hypertensive Dahl SS rats. Results of the present study show that T3 reduces vascular hypercontractility in aortas from hypertensive Dahl SS rats. This rapid-onset effect of T3 on vasomotor response has been previously observed by us and other groups (Hiroi et al., 2006; Samuel et al., 2017; Llevenes et al., 2018).

PKG plays an important role in maintenance of vascular smooth muscle phenotype and vascular relaxation (Choi et al., 2018). Activation of PKG in VSMC can lead to phosphorylation of VASP at serine 239, resulting in vascular relaxation (Holt et al., 2016). In the present study, we found decreased protein expression of PKG in aortas from Dahl SS rats, which was accompanied by reduced levels of phosphorylated VASP, indicating down-regulation of the PKG/VASP signaling pathway in hypertensive blood vessels. Importantly, T3 significantly increased levels of phosphorylated VASP in aortas from Dahl SS rats, and this effect was associated with reduced vascular hypercontractility. These findings are in accordance with our previous study (Samuel et al., 2017) in which we reported that T3 rapidly potentiates vascular relaxation via increased phosphorylation of VASP, which corresponds to a nongenomic, noncanonical effect of T3 (Bassett et al., 2003; Flamant et al., 2017). These new findings firmly establish that T3 is able to induce significant posttranslational modification of VASP in blood vessels to cause rapid changes in vascular reactivity and support an important vasoactive role.

Oxidative stress is another key component playing a role in the pathogenesis of hypertensive vascular dysfunction (Touyz, 2004). Damage from oxidative stress has been shown to cause impaired vasoconstriction and vasodilatation (Rodrigo et al., 2003; Paravicini and Touyz, 2006). Consistent with these previous findings, we also found that vascular dysfunction in hypertensive Dahl SS rats is accompanied by augmented vascular oxidative stress, which was markedly reduced
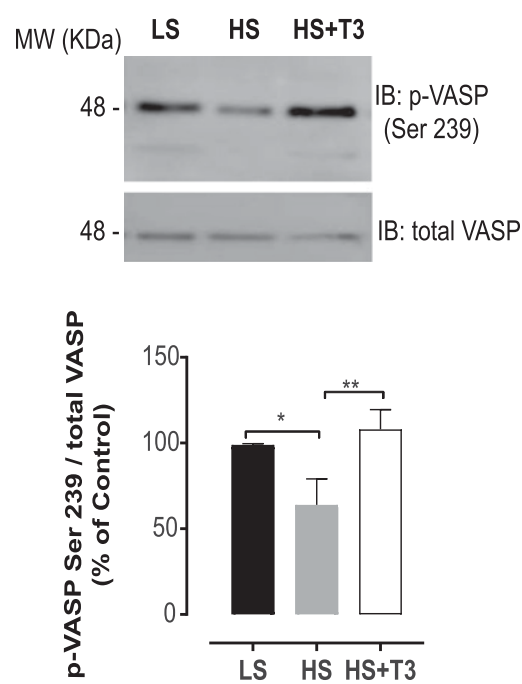

Fig. 6. T3 increased phosphorylation of VASP in hypertensive aortas. Aortas were treated with $0.1 \mu \mathrm{M}$ T3 for 30 minutes. After treatment, levels of phosphorylated VASP in the serine 239 were assessed using Western blot analysis. Top panel: representative immunoblots. Bottom panel: quantification of intensity of bands. Data are expressed as mean \pm S.E.M., $n=7$ per group, $* P<0.05$ vs. LS group, ${ }^{*} * P<0.05$ vs. HS group.

by short-term T3 treatment. These data support the hypothesis that reduction of vascular ROS levels and subsequent increase in nitric oxide (NO) bioavailability caused by T3 may be a mechanism contributing, at least in part, to the reduced vasoconstriction as well as the increased endothelium-dependent vasodilation observed in aortas from hypertensive Dahl SS rats directly treated with T3.

This antioxidant effect of T3 is in accordance with others studies that have suggested T3 as a potential antioxidant agent. For example, thyroid hormones have free radical scavenging capacity and are able to reduce low-density lipoprotein oxidation in vitro (Oziol et al., 2001). Moreover, considering that oxidative stress also plays a key role in renal damage in Dahl SS rats (De Miguel et al., 2011), further studies investigating the effects of T3 treatment in kidneys of hypertensive animals may expand the benefits of T3 to renal microvasculature. Moreover, future studies will be designed to examine the mechanisms by which T3 reduces vascular oxidative stress.

Although we cannot account in the present study for T3 causing endothelium-independent vasodilation in hypertensive blood vessels, our data imply that T3 activates endothelial cells (acetylcholine curve data, Fig. 3), resulting in NO release that stimulates cGMP production and activates PKG, which consequently phosphorylates VASP (Western blot data, Fig. 6) and causes smooth muscle cell relaxation. Moreover, T3 significantly reduced vascular oxidative stress, implying an increase in vascular NO bioavailability due to reduced ROS levels. Therefore, it is likely that the antioxidant effect of $\mathrm{T} 3$ also contributes to it vasodilator effect.

In conclusion, our data demonstrate that T3 not only plays a role in promoting vasodilation but also decreases vasoconstriction in hypertensive vasculature, most likely via a combination of endothelial and VSMC-related mechanisms.

The therapeutic use of $\mathrm{T} 3$ in treating cardiovascular disorders has been under continued debate (Gerdes and 

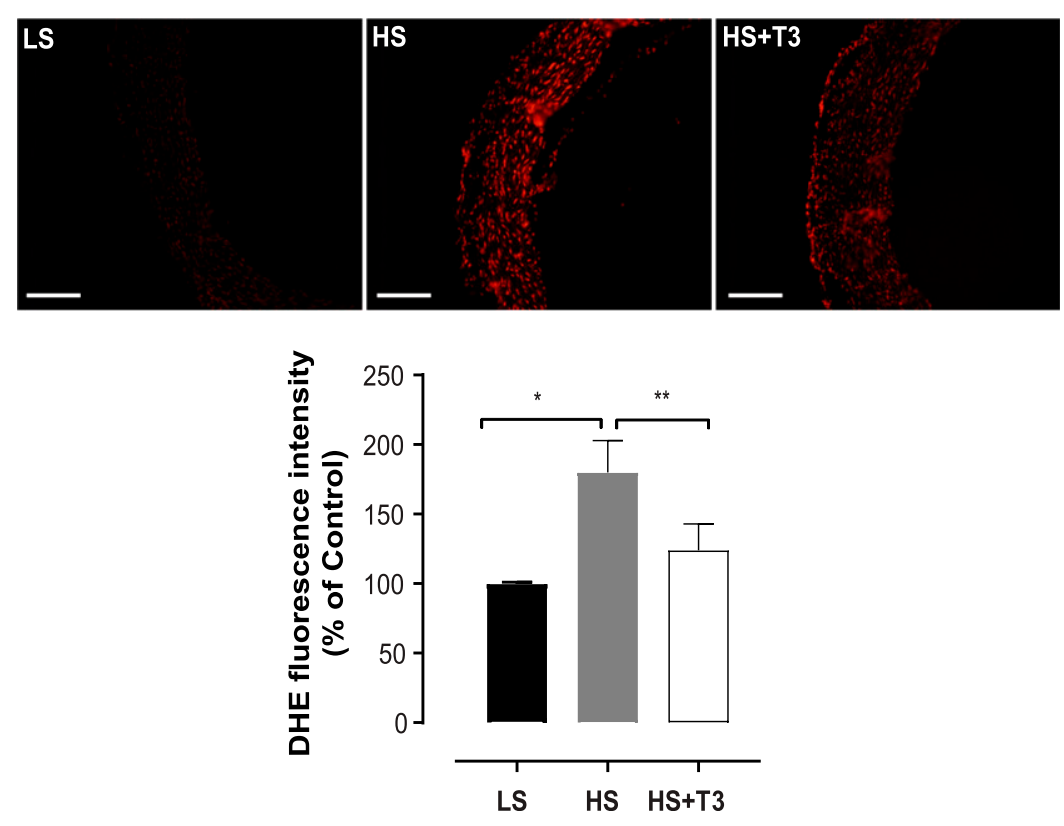

Iervasi, 2010). Our findings offer further evidence that T3 exerts a protective effect in the cardiovascular system. Improved understanding of cross-talk between endothelial and VSMC-related mechanisms by which T3 regulates vascular function may open new avenues of research and treatment options for vascular dysfunction found in cardiovascular disease. The present study was not intended to examine $\mathrm{T} 3$ as a potential agent reducing blood pressure but specifically examined the short-term direct effects of T3 in blood vessels from hypertensive rats. In fact, in vivo treatment with T3 did not show an effect on blood pressure in the Dahl salt-sensitive rat model, which was expected because the regulation of blood pressure in this model is mostly attributed to renin angiotensin system and renal injury mechanisms. Rapid vascular effects of T3 without affecting blood pressure have also been observed in human studies. Hamilton et al. (1998) showed that short-term intravenous administration of T3 in humans caused peripheral vasodilatory effects and reduced systemic vascular resistance without causing changes in blood pressure.

Although serum T3 levels were normal, our results imply impaired thyroid hormone signaling in vessels from Dahl SS rats. Our group previously reported that low cardiac tissue T3 levels were related to reduced cardiac function in diabetic rats despite normal serum thyroid hormone levels (Weltman et al., 2014). Our data suggest that thyroid hormone bioavailability may also be altered in vascular tissue despite normal serum hormone levels, which merits further investigation.

Despite advances in antihypertensive drug therapy in recent decades allowing significantly prolonged life spans in hypertensive patients, some patients do not benefit from these medications (Mozaffarian et al., 2015). This indicates that additional vasoactive agents are needed to attenuate the vascular complications that negatively impact hypertensive individuals. Our data do not postulate T3 as a treatment of high blood pressure but do raise the interesting possibility that T3 can directly ameliorate vascular dysfunction via both endothelial and VSMC-related mechanisms,
Fig. 7. T3 decreased oxidative stress in hypertensive aortas. Aortas from the experimental groups were either treated or not treated with $0.1 \mu \mathrm{M}$ T3 for 30 minutes followed by detection of oxidative stress using DHE probe. Top panel: representative photomicrographs depicting DHE (red fluorescence) in aortas. Bottom panel: quantification of fluorescent intensity in each aorta cross-section. Data are expressed as mean \pm S.E.M., $n=7$ per group, $* P<0.05$ vs. LS group, ${ }^{* *} P<0.05$ vs. HS group. Scale bar, $100 \mu \mathrm{m}$. which represents a critical preclinical step in the development of hypertension.

In conclusion, our results indicate that T3 mitigates vascular dysfunction in hypertensive Dahl SS rats via an antioxidant effect and activation of the PKG/VASP signaling pathway, increasing our knowledge of the mechanisms of nongenomic effects of T3 in the vascular system.

\section{Authorship Contributions}

Participated in research design: Carrillo-Sepulveda, Maracheril, Gerdes.

Conducted experiments: Carrillo-Sepulveda, Panackal, Maracheril, Maddie, Patel, Ojamaa.

Performed data analysis: Carrillo-Sepulveda, Panackal, Maddie, Ojamaa, Savinova, Gerdes.

Wrote or contributed to the writing of the manuscript: CarrilloSepulveda, Panackal, Maracheril, Maddie, Ojamaa, Savinova, Gerdes.

\section{References}

Bassett JH, Harvey CB, and Williams GR (2003) Mechanisms of thyroid hormone receptor-specific nuclear and extra nuclear actions. Mol Cell Endocrinol 213:1-11.

Cai Y, Manio MM, Leung GP, Xu A, Tang EH, and Vanhoutte PM (2015) Thyroid hormone affects both endothelial and vascular smooth muscle cells in rat arteries. Eur $J$ Pharmacol 747:18-28.

Carrillo-Sepúlveda MA, Ceravolo GS, Furstenau CR, Monteiro P, Bruno-Fortes Z, Carvalho MH, Laurindo FR, Tostes RC, Webb RC, and Barreto-Chaves ML (2013) Emerging role of angiotensin type 2 receptor (AT2R)/Akt/NO pathway in vascular smooth muscle cell in the hyperthyroidism. PLoS One 8:e61982.

Carrillo-Sepulveda MA, Spitler K, Pandey D, Berkowitz DE, and Matsumoto T (2015) Inhibition of TLR4 attenuates vascular dysfunction and oxidative stress in diabetic rats. J Mol Med (Berl) 93:1341-1354.

Choi S, Park M, Kim J, Park W, Kim S, Lee DK, Hwang JY, Choe J, Won MH, Ryoo S et al. (2018) TNF- $\alpha$ elicits phenotypic and functional alterations of vascular smooth muscle cells by miR-155-5p-dependent down-regulation of cGMP-dependent kinase 1. J Biol Chem 293:14812-14822.

De Miguel C, Guo C, Lund H, Feng D, and Mattson DL (2011) Infiltrating T lymphocytes in the kidney increase oxidative stress and participate in the development of hypertension and renal disease. Am J Physiol Renal Physiol 300:F734-F742.

Drenjancevic-Peric I and Lombard JH (2004) Introgression of chromosome 13 in Dah salt-sensitive genetic background restores cerebral vascular relaxation. Am J Physiol Heart Circ Physiol 287:H957-H962

Flamant F, Cheng SY, Hollenberg AN, Moeller LC, Samarut J, Wondisford FE, Yen PM, and Refetoff S (2017) Thyroid hormone signaling pathways: time for a more precise nomenclature. Endocrinology 158:2052-2057.

Gerdes AM and Iervasi G (2010) Thyroid replacement therapy and heart failure. Circulation 122:385-393. 
Gómez-Roso M, Montero MJ, Carrón R, and Sevilla MA (2009) Cardiovascular changes in spontaneously hypertensive rats are improved by chronic treatment with zofenopril. Br J Pharmacol 158:1911-1921.

Gu Y, Zheng L, Zhang Q, Liu L, Meng G, Yao Z, Wu H, Xia Y, Bao X, Shi H, et al. (2018) Relationship between thyroid function and elevated blood pressure in euthyroid adults. J Clin Hypertens (Greenwich) 20:1541-1549.

Hamilton MA, Stevenson LW, Fonarow GC, Steimle A, Goldhaber JI, Child JS, Chopra IJ, Moriguchi JD, and Hage A (1998) Safety and hemodynamic effects of intravenous triiodothyronine in advanced congestive heart failure. Am $J$ Cardiol 81:443-447.

Hiroi Y, Kim HH, Ying H, Furuya F, Huang Z, Simoncini T, Noma K, Ueki K, Nguyen NH, Scanlan TS, et al. (2006) Rapid nongenomic actions of thyroid hormone. Proc Natl Acad Sci USA 103:14104-14109.

Holt AW, Martin DN, Shaver PR, Adderley SP, Stone JD, Joshi CN, Francisco JT, Lust RM, Weidner DA, Shewchuk BM, et al. (2016) Soluble guanylyl cyclaseactivated cyclic GMP-dependent protein kinase inhibits arterial smooth muscle cell migration independent of VASP-serine 239 phosphorylation. Cell Signal 28: 1364-1379

Khorshidi-Behzadi M, Alimoradi H, Haghjoo-Javanmard S, Reza Sharifi M, Rahimi N, and Dehpour AR (2013) The effect of chronic hyperthyroidism and restored euthyroid state by methimazole therapy in rat small mesenteric arteries. Eur J Pharmacol 701:20-26.

Kisso B, Patel A, Redetzke R, and Gerdes AM (2008) Effect of low thyroid function on cardiac structure and function in spontaneously hypertensive heart failure rats. $J$ Card Fail 14:167-171.

Liu KL, Lo M, Canaple L, Gauthier K, Del Carmine P, and Beylot M (2014) Vascular function of the mesenteric artery isolated from thyroid hormone receptor-a knockout mice. J Vasc Res 51:350-359.

Llévenes P, Balfagón G, and Blanco-Rivero J (2018) Thyroid hormones affect nitrergic innervation function in rat mesenteric artery: role of the PI3K/AKT pathway. Vascul Pharmacol 108:36-45.

Lozano-Cuenca J, Lopez-Canales OA, Aguilar-Carrasco JC, Villagrana-Zesati JR, Lopez-Mayorga RM, Castillo-Henkel EF, and Lopez-Canales JS (2016) Pharmacological study of the mechanisms involved in the vasodilator effect produced by the acute application of triiodothyronine to rat aortic rings. Braz J Med Biol Res 49:e5304.

Lüscher TF, Raij L, and Vanhoutte PM (1987) Endothelium-dependent vascular responses in normotensive and hypertensive Dahl rats. Hypertension 9 157-163.

Maia AR, Batista TM, Victorio JA, Clerici SP, Delbin MA, Carneiro EM, and Davel AP (2014) Taurine supplementation reduces blood pressure and prevents endothelial dysfunction and oxidative stress in post-weaning protein-restricted rats. PLoS One 9:e105851.

Mancia G and Giannattasio C (2015) Diagnostic and therapeutic problems of isolated systolic hypertension. J Hypertens 33:33-43.

Mordi I, Mordi N, Delles C, and Tzemos N (2016) Endothelial dysfunction in human essential hypertension. J Hypertens 34:1464-1472.

Mozaffarian D, Benjamin EJ, Go AS, Arnett DK, Blaha MJ, Cushman M, de Ferranti S, Després JP, Fullerton HJ, Howard VJ, et al.; American Heart Association Statistics Committee and Stroke Statistics Subcommittee (2015) Heart disease and stroke statistics-2015 update: a report from the American Heart Association [published corrections appear in Circulation (2015) 131:e535 and (2016) 133:e417]. Circulation 131:e29-e322.
Ojamaa K, Balkman C, and Klein IL (1993) Acute effects of triiodothyronine on arterial smooth muscle cells. Ann Thorac Surg 56 (1 Suppl):S61-S66, discussion S66-S67.

Ojamaa K, Klemperer JD, and Klein I (1996) Acute effects of thyroid hormone on vascular smooth muscle. Thyroid 6:505-512.

Oziol L, Faure P, Vergely C, Rochette L, Artur Y, Chomard P, and Chomard P (2001) In vitro free radical scavenging capacity of thyroid hormones and structural analogues. J Endocrinol 170:197-206.

Paravicini TM and Touyz RM (2006) Redox signaling in hypertension. Cardiovasc Res 71:247-258.

Park KW, Dai HB, Ojamaa K, Lowenstein E, Klein I, and Sellke FW (1997) The direct vasomotor effect of thyroid hormones on rat skeletal muscle resistance arteries. Anesth Analg 85:734-738.

Polat Canbolat I, Belen E, Bayyigit A, Helvaci A, and Kilickesmez K (2017) Evaluation of daily blood pressure alteration in subclinical hypothyroidism. Acta Cardiol Sin 33:489-494.

Rapp JP (1982) Dahl salt-susceptible and salt-resistant rats. A review. Hypertension 4:753-763.

Razvi S, Jabbar A, Pingitore A, Danzi S, Biondi B, Klein I, Peeters R, Zaman A, and Iervasi G (2018) Thyroid hormones and cardiovascular function and diseases. $J$ Am Coll Cardiol 71:1781-1796.

Rodrigo R, Passalacqua W, Araya J, Orellana M, and Rivera G (2003) Implications of oxidative stress and homocysteine in the pathophysiology of essential hypertension. J Cardiovasc Pharmacol 42:453-461.

Samuel S, Zhang K, Tang YD, Gerdes AM, and Carrillo-Sepulveda MA (2017) Triiodothyronine potentiates vasorelaxation via PKG/VASP signaling in vascular smooth muscle cells. Cell Physiol Biochem 41:1894-1904.

Sullivan JM (1991) Salt sensitivity. Definition, conception, methodology, and longterm issues. Hypertension 17 (1 Suppl):I61-I68.

Teran FJ, Johnson RA, Stevenson BK, Peyton KJ, Jackson KE, Appleton SD, Durante W, and Johnson FK (2005) Heme oxygenase-derived carbon monoxide promotes arteriolar endothelial dysfunction and contributes to salt-induced hypertension in Dahl salt-sensitive rats. Am J Physiol Regul Integr Comp Physiol 288:R615-R622.

Touyz RM (2004) Reactive oxygen species, vascular oxidative stress, and redox signaling in hypertension: what is the clinical significance? Hypertension 44:248-252.

Touyz RM and Schiffrin EL (2004) Reactive oxygen species in vascular biology: implications in hypertension. Histochem Cell Biol 122:339-352.

Weltman NY, Ojamaa K, Schlenker EH, Chen YF, Zucchi R, Saba A, Colligiani D, Rajagopalan V, Pol CJ, and Gerdes AM (2014) Low-dose $\mathrm{T}_{3}$ replacement restores depressed cardiac $\mathrm{T}_{3}$ levels, preserves coronary microvasculature and attenuates cardiac dysfunction in experimental diabetes mellitus. Mol Med 20:302-312.

Weltman NY, Pol CJ, Zhang Y, Wang Y, Koder A, Raza S, Zucchi R, Saba A, Colligiani D, and Gerdes AM (2015) Long-term physiological T3 supplementation in hypertensive heart disease in rats. Am $J$ Physiol Heart Circ Physiol 309: H1059-H1065.

Address correspondence to: Dr. Maria Alicia Carrillo-Sepulveda, Department of Biomedical Sciences, New York Institute of Technology College of Osteopathic Medicine, Northern Boulevard, Old Westbury, NY 11568. E-mail: mcarrill@nyit.edu or aliciasepulveda10@gmail.com 